PROYECC I DNES

ricvista de Matemática Vol, lo Pág. 53-57

1991 - ISSN 0716-0917

Univer sidad católica dẹ l Nor te

mitafagasta - Chile

\title{
A FIXED POINT THEOREM FOR ORBITALLY CONTINUOUS FUNCTIONS.
}

Ioamis K. Argyros

\section{ABSTRACT}

A fixed point theorem for orbitally contimoug func tions is presented which extends well knoum results.

\section{INTRODUCTION}

We extend a result of ,]aggi [4] for orbitally continunus functions. Note that ciric [2] has shown that orbitally continuous functions need not be continuous.

Definition 1 .

Let $x$ be a normed 1 inear space and $P$ be a mapping of $x$ into itself. Then $P$ is said to be asymptotically regular at a point $q E x$ if

$$
\left\|P_{q}^{n}-P^{n+1} q\right\| \rightarrow 0, \text { as } n \rightarrow \infty \text {. }
$$


Denote by, $P / \bar{A}(q, P)$ the restriction of $P$ to $\bar{A}(q, P)$, the closure of the orbit of $P$ at $q[4]$.

Definition 2 .

A mapping of $P$ defined on $X$ into itself is said to be $q$-orbitally continuous if

$$
P / \bar{A}(q, P): \bar{A}(q, P)+X
$$

is continuous.

We now prove the theorem.

\section{Theorem}

Let $S$ be a convex subset of a normed linear space $X$ such that $S t \phi$, let $P: S \rightarrow S$ be a mapping satisfying

$$
\begin{aligned}
\|P x-P y\| & \leqslant c_{1}(x, y)\|x-y\|+c_{2}(x, y)[\|x-P x\|+\|y-P y\|] \\
& +c_{3}(x, y)[\|x-P y\|+\|y-P x\|]+ \\
& +c_{4}(x, y)\|y-P x\|\left[\frac{1+\|y-P y\|+\|x-P y\|]}{1+\| x}+\right. \\
& +c_{5}(x, y)\|x-P y\| \frac{[1+\|x-P x\|+\|y-P x\|]}{1+\|x-y\|} \| x
\end{aligned}
$$

for all $x, y \in S$ and $c_{i}: S \times S+[0,1[$ with

$\sup _{x, y \in S}\left\{c_{1}(x, y)+2 c_{2}(x, y)+2 c_{3}(x, y)+c_{4}(x, y)+3 c_{5}(x, y)\right\}=1$

$\inf _{x, y \in S} c_{2}(x, y)>0$

Let us choose $\lambda_{k}, i=0,1,2, \ldots, k$ for some $k \in N$, $\left.\lambda_{i} \in\right] 0,1\left[\right.$, such that $\sum_{i=0} \lambda_{i}=1$. Define the operator $B$ on $S$ such that

$B-\sum_{i=0}^{k} \lambda_{i} P^{i}, p^{0}$ being the identity mapping on $S$. 
Assume :

(i) there exists a point $q \in S$ such that $w \in S$ is a cluster point of the sequence $\left\{B^{n} q\right\}, n=1,2, \ldots \ldots$,

(ii) $B$ is q-orbitally continuous and asymptotically regular at $q$ and

(iii) the function $c_{5}$ is such that

$c_{5}(q, y)=0$ for all yES.

Then the sequence $\left\{B^{n} q\right\}, n=1,2, \ldots$ converges to $w$ and $w$ is the unique fixed point of $p$ on $S$.

\section{Proof. Step 1. Existence of the fixed point}

By hypothesis (i), there exists a subsequence $\left\{B^{n} i q, i=1,2, \ldots\right.$ of the sequence $\left\{B^{n} q\right\}$ converging to $w$. By hypothesis (ii), we have

$$
(I-B) B^{n} i q \rightarrow(I-B) w
$$

and

$$
\left\|(I-B) B^{n} q\right\|=\left\|B^{n} q-B^{n_{i}+1} q\right\|+0 \text { as } i \rightarrow \infty
$$

Sn, $\|(I-B) w\|=0 \rightarrow B w=w$ and $P w=w$.

\section{Step 2. Uniqueness}

Let $v$ he such that $v \in S, v \neq w$ and $\Gamma v \cdot v$. By hypothesis

(i), we have

$$
\begin{aligned}
& \|w-v\|=\|P w-P v\| \leqslant c_{1}(w, v)\|w-v\|+c_{2}(w, v)[\|w-P w\|+\|v-P v\|]+ \\
& +c_{3}(w, v)[\|w-P v\|+\|v-P w\|]+c_{4}(w, v)\|v-P w\|[1+\|v-P v\|+\|w-P v\|] \\
& +c_{5}(w, v)\|w-P v\| \frac{[1+\|w-P w\|+\|v-P w\|]}{1+\|w-v\|} \\
& \leqslant\left\{c_{1}(w, v)+2 c_{3}(w, v)+c_{4}(w, v)+c_{5}(w, v)\right\}\|w-v\| \\
& <\|w-v\|
\end{aligned}
$$


which is a contradiction $50 \quad w=v$.

Step 3. The convergence of the sequence $\left\{B^{n} q\right\}, n=1,2, \ldots$

(i)

Let $v$ be an arbitrary element of $S$. We have using hypothesis

$$
\begin{aligned}
& \|P w-P v\| \leqslant c_{1}(w, v)\|w-v\|+c_{2}(w, v)\|v-P v\| \\
& +c_{3}(w, v)\{\|w-v\|+\|w-P v\|]+c_{4}(w, v)\|w-P v\|+ \\
& +c_{5}(w, v)\|v-P w\| \frac{[1+\|v-P v\|+\|w-P v\|]}{1+\| v}-w \|
\end{aligned}
$$

which gives iusing the triangle inequality and the fact that

$$
\|v-w\|<1+\|v-w\|
$$

$\|P v-P w\| \leqslant \frac{c_{1}(w, v)+c_{2}(w, v)+c_{3}(w, v)}{1-c_{2}(w, v)-c_{3}(w, v)-c_{4}(w, v)-2 c_{5}(w, v)}\left(\|w-v\|+c_{5}(w, v)\right)$.

We can prove similarly, for $i=1,2, \ldots, k$,

$\left\|p^{i} v-p^{i} w\right\| \leqslant\|w-v\|+\frac{c_{5}(w, v)}{1-c_{2}(w, v)-c_{3}(w, v)-c_{4}(w, v)-\overline{2} c_{5}(w, v)}$

We now have that

$$
\begin{aligned}
\|B v-w\| & =\| \sum_{i=0}^{k} \lambda_{i} P^{i}\left(v-w\left\|\leqslant \sum_{i=0}^{k} \lambda_{j}\right\| p^{i} v-p^{i} w \|\right. \\
& \leqslant \sum_{i=0}^{k} \lambda_{i}(\|v-w\|+f(w, v)) \\
& \leqslant\|v-w\|+f(w, v)
\end{aligned}
$$

where,

$$
f(w, v)=\frac{c_{5}(w, v)}{1-c_{2}(w, v)-c_{3}(w, v)-c_{4}(w, v)-2 c_{5}(w, v)}
$$

In particular, the above inequality for $v=q$ gives,

$$
\left\|B^{n+1} q-w\right\| \leqslant\left\|B^{n} q-w\right\| .
$$




\section{Since $B^{n_{i}} q+w$ as $i+\infty$ this implies that $B^{n} q \rightarrow w$ as $n+\infty$. The case $v=w$ trivially gives us $B^{n} v \rightarrow w$ and tirat completes} the proof.

Note that if we take $c_{1}=c_{3}=c_{4}=c_{5}-0, c_{2}=\frac{1}{2}$ and $k=1$ we obtain the result in [4].

\section{REFERENCES}

[1] BOYD, D.W. and WONG, J.S.W. On nonlinear contractions, Proc. Amer. Math. Soc., 20, (190̃ ), 458-464.

[2] CIRIC, Lj. Fixed and periodic points of almost contractive operators, Mathematica Balcanica, 3, (1973), 33-39.

[3] ISTRACESCU, C. Fixed point theory, ACademic Press (1984).

[4] JAGGI, D.S. Fixed point theorems for orbitally continuous functions 1I, Indian J. Math. 19(2), (1977), 113-119.

[5] KHAN, M.S. Common fixed point theorems for multivalued mappings, $\mathrm{Pa-}$ cific J. Math. 95, (1981), 337-347.

\section{Ioanis K. Argyzos}

Department of Mathematics

New Mexico State University

L_as Cruces, NM 88003 